\title{
Effects of Temperature and Pressure on the Dielectric Constant in Non-Polar Polymers
}

\author{
Hiroyuki SASABe and Shogo SAIto \\ Materials Division, Electrotechnical Laboratory, \\ Tanashi, Tokyo, Japan. \\ (Received February 28, 1972)
}

\begin{abstract}
Changes of dielectric constant $\varepsilon$ in non-polar and slightly polar polymers with temperature and pressure have been analyzed in terms of the Clausius-Mossotti equation. It is concluded that the Clausius-Mossotti equation is valid for polyethylene but should be modified and expressed in the form $M(\varepsilon-1) / \rho(\varepsilon+2)=\left(4 \pi N_{0} / 3\right)$ $\times\left(\alpha+\mu^{2} / 3 k T\right)$ for slightly polar polymers. It is also concluded from the comparison of the polarizability $\alpha$ for low-molecular-weight substances with $\alpha$ for polymers that the determining factor for $\alpha$ for polymers is the molecular structure of the monomer unit. $\partial \varepsilon / \partial T$ and $\partial \varepsilon / \partial P$ change suddenly at a transition point such as glass transition $\left(T_{\mathrm{g}}\right)$ and phase transition in the crystalline region $\left(T_{\chi}\right)$, and $\mathrm{d} T_{\mathrm{g}} / \mathrm{d} P$ for polystyrene can be evaluated as $3.2 \times 10^{-2} \mathrm{~K}$ atm $^{-1}$ and $\mathrm{d} T_{\chi} / \mathrm{d} P$ for poly(tetrafluoroethylene) as $2.3 \times 10^{-2}$ $\mathrm{K}$ atm $^{-1}$.
\end{abstract}

KEY WORDS Dielectric Constant / Clausius-Mossotti Equation /

Polarizability / Glass Transition / Crystalline Phase Transition /

Polystyrene / Poly(tetrafluoroethylene) /

Studies on the dielectric properties of polymers as functions of temperature and pressure may be roughly divided into two categories: (1) dielectric relaxations associated with molecular motions of polymer chains and (2) limiting highand low-frequency dielectric constants of polymers. The former category (1) has been vigorously investigated over these last several years. ${ }^{1}$ According to those investigations it has been made clear that temperature and pressure dependences of dielectric relaxations in polar polymers can be interpreted by those of free volume, cofigurational entropy and/or intermolecular interactions. On the other hand, category (2) has not been sufficiently investigated as yet.

Studies on effects of temperature and pressure on limiting high-frequency dielecric constant $\varepsilon_{\infty}$ of polymers is of particular interest, since $\varepsilon_{\infty}$ is an important quantity for the evaluation of dielectric increments and is closely related to the optical refractive index. Non-polar polymers whose dielectric constant $\varepsilon$ is independent of frequency and corresponds to $\varepsilon_{\infty}$ in polar polymers are suitable for such a study. In this paper we will discuss the changes of $\varepsilon$ in nonpolar and slightly polar polymers such as poly- ethylene, poly(tetrafluoroethylene), polypropylene, and polystyrene, with temperature and pressure from the view-point of the molecular structure (that is, the check of the ClausiusMossotti equation), and their correlations with transition phenomena in polymers.

While non-polar dielectric materials have no permanent dipoles, electronic and atomic polarizations (or, more exactly, optical and infrared polarizations) are induced by the application of an external electric field. Contributions of these induced dipole moments to the dielectric constant are expressed by the well-known Clausius-Mossotti equation,

$$
C_{\mathrm{M}} \equiv \frac{\varepsilon-1}{\varepsilon+2} \frac{M}{\rho}=\frac{4 \pi}{3} N_{0} \alpha
$$

where $M$ is the molecular weight, $\rho$ the density, $N_{0}$ Avogadro's number, $\alpha$ the polarizability, and, in the case of polymers, $M$ is the molecular weight of a monomer unit and $\alpha$ is the polarizability of a monomer unit. In fact, $C_{\mathrm{M}}$ is not a constant and can be approximated by a polynomial in $\rho$. If $C_{\mathrm{M}}$ is expressed as $C_{\mathrm{M}}=a$ $+b \rho+\cdots$, then the factor $b \rho$ is about one-tenth of the factor $a$ for the $n$-alkane family. ${ }^{2}$ There- 


\section{H. SAsabe and S. Saito}

fore we assume that $C_{\mathrm{M}}$ is independent of $\rho$ for simplicity here, and take eq 1 as the fundamental equation for discussions of temperature and pressure dependences of $\varepsilon$ in non-polar polymers. In eq 1 both $\rho$ and $\alpha$ are considered to be functions of temperature $T$ and pressure $P$. However changes of $\alpha$ with $T$ and $P$ are expected to be much smaller than those of $\rho$ in the temperature and pressure ranges available, since they are caused by the distortions of electron clouds surrounding the atomic nuclei.

For slightly polar polymers eq 1 , namely $C_{\mathrm{M}}$, is modified to

$$
C_{\mathrm{M}} \equiv \frac{\varepsilon-1}{\varepsilon+2} \frac{M .}{\rho}=\frac{4 \pi N_{0}}{3}\left(\alpha+\frac{\mu^{2}}{3 k T}\right)
$$

if it is assumed that the polar groups can rotate freely in the liquid state. Here $\mu$ is the effective dipole moment of a monomer unit. We will check the validity of eq 1 and 2 from changes of $\rho$ with $T$ and $P$.

\section{EXPERIMENTAL}

Five polymer samples were used for this investigation: low-density polyethylene(PE-L), high-density polyethylene (PE-H), polypropylene (PP), poly(tetrafluoroethylene) (PTFE) and polystyrene (PS). In PE-L and PE-H, their monomer units do not have any permanent dipole, so long as their chains take a planar zigzag conformation. However a monomer unit in a helical chain, which is expected in PTFE, would have a small permanent dipole. PP and PS may not be suitable samples for the present purpose, since they are slightly polar.

The dielectric constant $\varepsilon$ was measured at 1 $\mathrm{kHz}$, using the GR 1620-A Capacitance Bridge Assembly (General Radio Co.). In order to analyze the observed data it was necessary to know temperature and pressure dependences of the specific volume of the polymers. Thermal expansion of polymers (at $1 \mathrm{~atm}$ ) was measured by means of the floatation (Archimedes) method. We used data for the isothermal compressibility of PE-L, PE-H, and PS obtained by Hellwege, Knappe, and Lehmann, ${ }^{3}$ of PTFE obtained by Weir ${ }^{4}$, and of PP obtained by Foster, Waldman, and Griskey. ${ }^{5}$

The dielectric constant $\varepsilon$ of a polymer sample was calculated from observed capacitance $C[\mathrm{pF}]$, according to the relation

$$
\varepsilon=C \times \frac{3.6 \pi t}{A}
$$

where $t[\mathrm{~cm}]$ is a film thickness of a polymer sample and $A\left[\mathrm{~cm}^{2}\right]$ is the effective electrode area which comprises the condenser. For the calculation of $\varepsilon$, we must correct for changes of the geometrical capacitance, since both $t$ and $A$ changes with $T$ and $P$. Under the conditions of the isotropic compression and expansion of the sample, $\varepsilon$ at $T$ and $P, \varepsilon(T, P)$, is given as

$$
\begin{aligned}
\varepsilon(T, P)= & C(T, P) \\
& \times \frac{3.6 \pi t_{\mathrm{r}}}{A} \frac{1+\alpha_{1}\left(T-T_{\mathrm{r}}\right)-\beta_{1}\left(\boldsymbol{P}-\boldsymbol{P}_{\mathrm{r}}\right)}{1+2 \alpha_{1}\left(T-T_{\mathrm{r}}\right)-2 \beta_{1}\left(\boldsymbol{P}-\boldsymbol{P}_{\mathrm{r}}\right)}
\end{aligned}
$$



Figure 1. Temperature dependence of $\varepsilon$ in PE-L, $\mathrm{PE}-\mathrm{H}$, and PP.

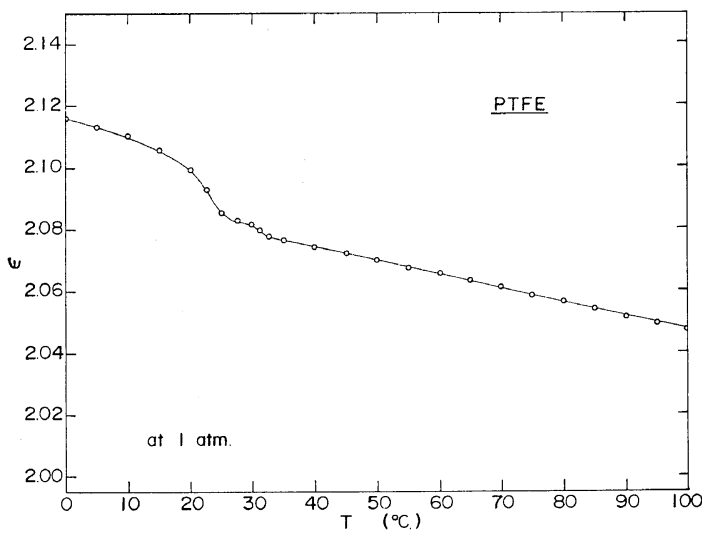

Figure 2. Temperature dependence of $\varepsilon$ in PTFE. 
Effects of $T$ and $P$ on $\varepsilon$ in Non-Polar Polymers

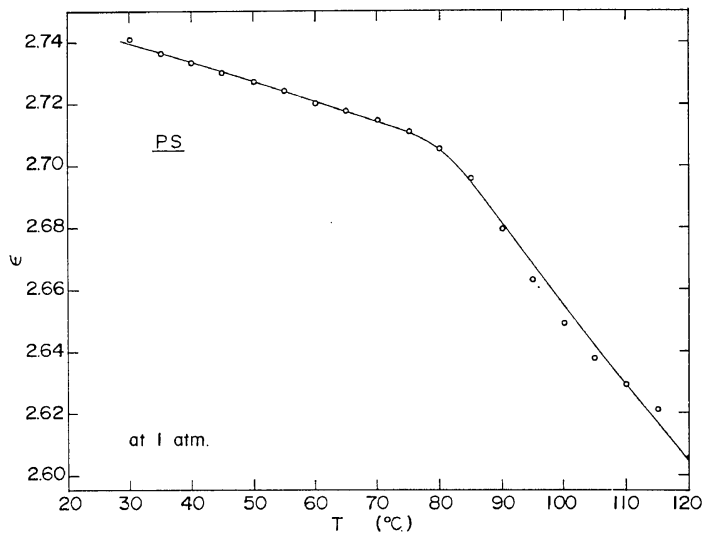

Figure 3. Temperature dependence of $\varepsilon$ in PS.



Figure 4. Pressure dependence of $\varepsilon$ in PE-L at fixed temperatures.

where $t_{\mathrm{r}}$ and $A_{\mathrm{r}}$ are respectively $t$ and $A$ at a reference temperature $T_{\mathrm{r}}$ and reference pressure $\boldsymbol{P}_{\mathrm{r}}, \alpha_{1}$, and $\beta_{1}$ are respectively the linear expansion coefficient and linear compressibility of the polymer.

Figure 1 shows temperature dependence of $\varepsilon$ in PE-L, PE-H, and PP at 1 atm. Figures 2 and 3 also show the effect of temperature on $\varepsilon$ of PTFE and PS, respectively. $\varepsilon$ decreases with temperature for all samples.

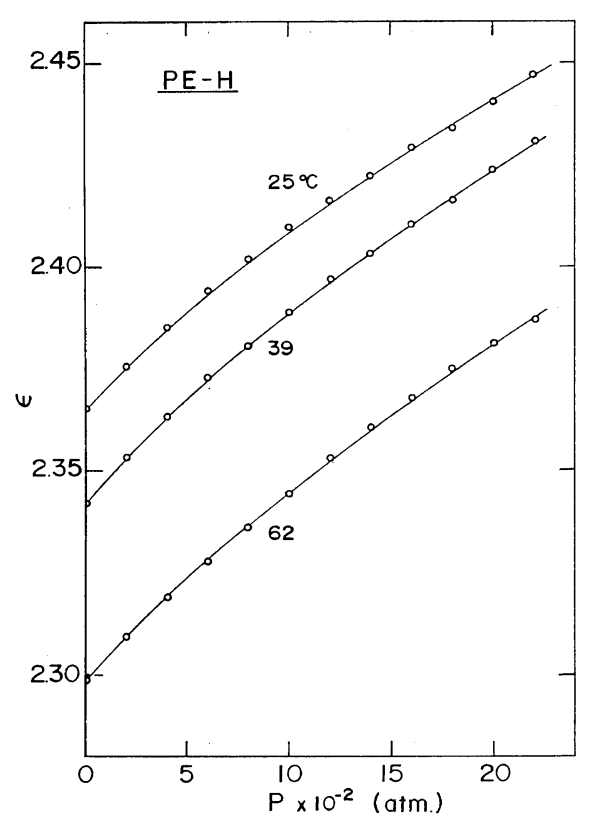

Figure 5. Pressure dependence of $\varepsilon$ in PE-H at fixed temperatures.

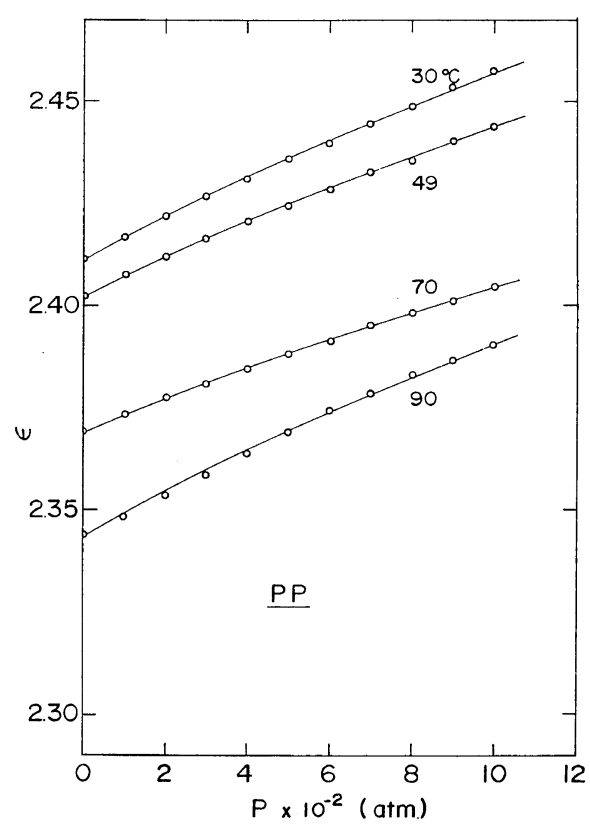

Figure 6. Pressure dependence of $\varepsilon$ in PP at fixed temperatures. 


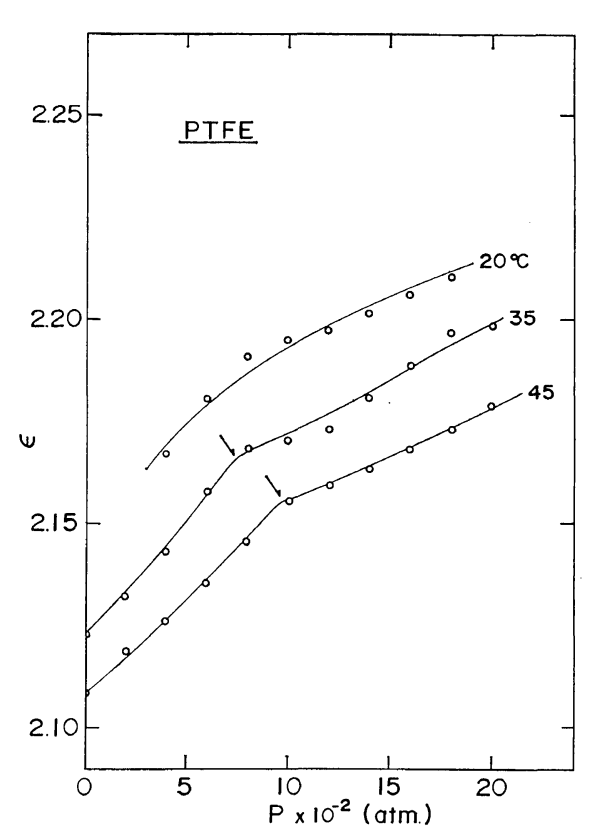

Figure 7. Pressure dependence of $\varepsilon$ in PTFE at fixed temperatnres.

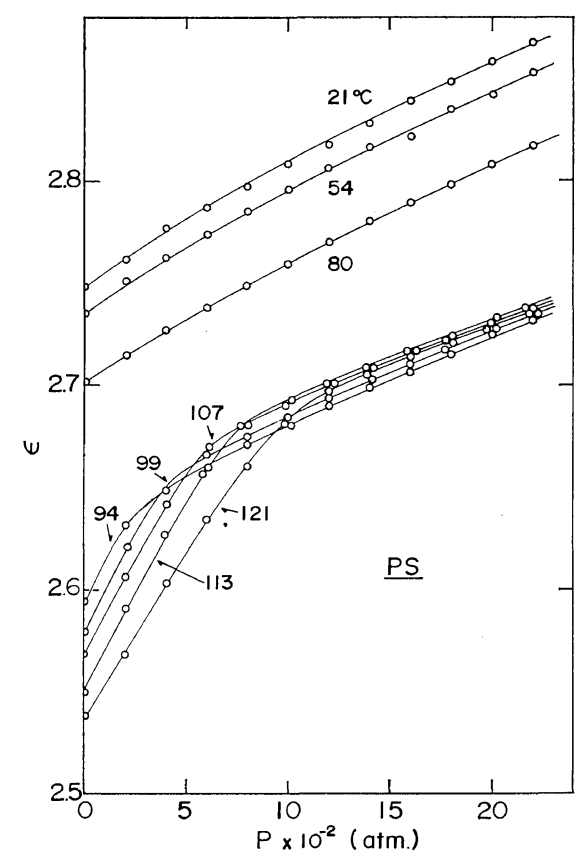

Figure 8. Pressure dependence of $\varepsilon$ in PS at fixed temperatures.
Figures $4 \sim 8$ show pressure dependences of $\varepsilon$ of PE-L, PE-H, PP, PTFE, and PS, respectively. $\varepsilon$ increases with pressure for all samples. Sudden changes of $\partial \varepsilon / \partial T$ or $\partial \varepsilon / \partial P$ are observed in the temperature or pressure dependence of $\varepsilon$ (Figures 1, 2, 3, 7, and 8). This would correspond to the transition phenomenon in the polymers, such as the glass transition, the melting or the phase transition in crystalline regions.

\section{DISCUSSION}

\section{Validity of Clausius-Mossotti Equation}

In order to check the appropriateness of the Clausius-Mossotti equation, values of $C_{\mathrm{M}}$ for various samples were plotted against $1 / T$ and $\boldsymbol{P}$. These results are shown in Figure 9. Changes of $C_{\mathrm{M}}$ for PE-L, PE-H, and PTFE with temperature are very small, as shown in Figure 9. $C_{\mathrm{M}}$ for PS at low temperatures (below the glass transition temperature $T_{\mathrm{g}}$ ), where permanent dipoles in PS do not rotate, also hardly changes with $1 / T$. These results suggest that variation of $\varepsilon$ with temperature for these polymers is governed by variation of $\rho$. On the contrary, $C_{\mathrm{M}}$ for PS at high temperatures (above $T_{\mathrm{g}}$ ) and PP changes almost linearly with $1 / T$. This variation is mostly affected by rotation of permanent dipoles. The dipole moment from the slope of $C_{\mathrm{M}} v s .1 / T$ plot by use of eq 2 are 0.27 and $0.50 \mathrm{D}$ for $\mathrm{PP}$ and PS, respectively. The calculated values agree with $\mu$ for the corresponding low-molecular-weight substances; $\mu$ for isobutane $(0.2 \mathrm{D})$ and ethyl-benzene $(0.58 \mathrm{D}) .{ }^{6}$ Figure 9 indicates that the contribution of $\mu$ to $C_{\mathrm{M}}$ is definitely smaller than that of $\alpha$, that is, $\left(\mu^{2} / 3 k\right)(1 / T)$ is much smaller than $\alpha$. Therefore $C_{\mathrm{M}}$ for the present samples is mostly affected by $\alpha$.

Figure 9 also shows that $C_{\mathrm{M}}$ hardly changes with $P$ at least for PE-L, PE-H, PTFE, and PP. This result supports the concept that the variation of $\varepsilon$ with $P$ is governed by variation of $\rho$. The dominant role of $\rho$ in the variation of $\varepsilon$ with $T$ or $P$ is demonstrated by Table I. If we differentiate eq 1 with respect to $T$ or $P$,

$$
\frac{\mathrm{d} \varepsilon}{\mathrm{d} X}=\frac{(\varepsilon-1)(\varepsilon+2)}{3}\left[\frac{\mathrm{d}(\ln \alpha)}{\mathrm{d} X}-\frac{\mathrm{d}(\ln V)}{\mathrm{d} X}\right]
$$

where $X$ is $T$ or $P$ and $V$ is the specific volume 
Effects of $T$ and $P$ on $\varepsilon$ in Non-Polar Polymers

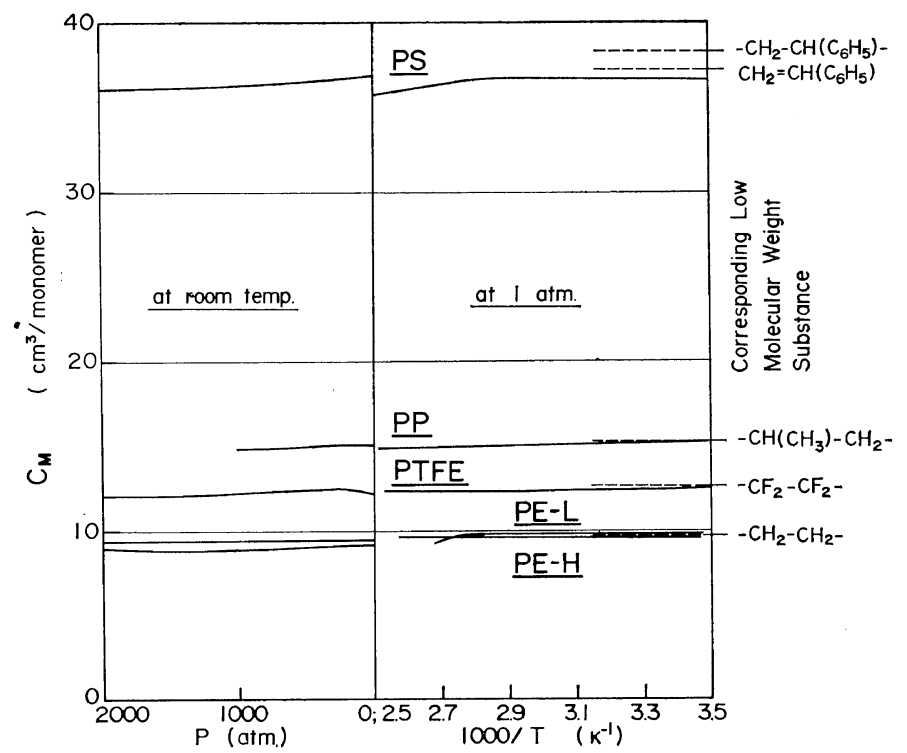

Figure 9. Comparison of values of $C_{\mathrm{M}}$ for polymers with those for low-molecularweight substances.

Table I. Average contribution of variation in volume to temperature and pressure dependence of dielectric constant

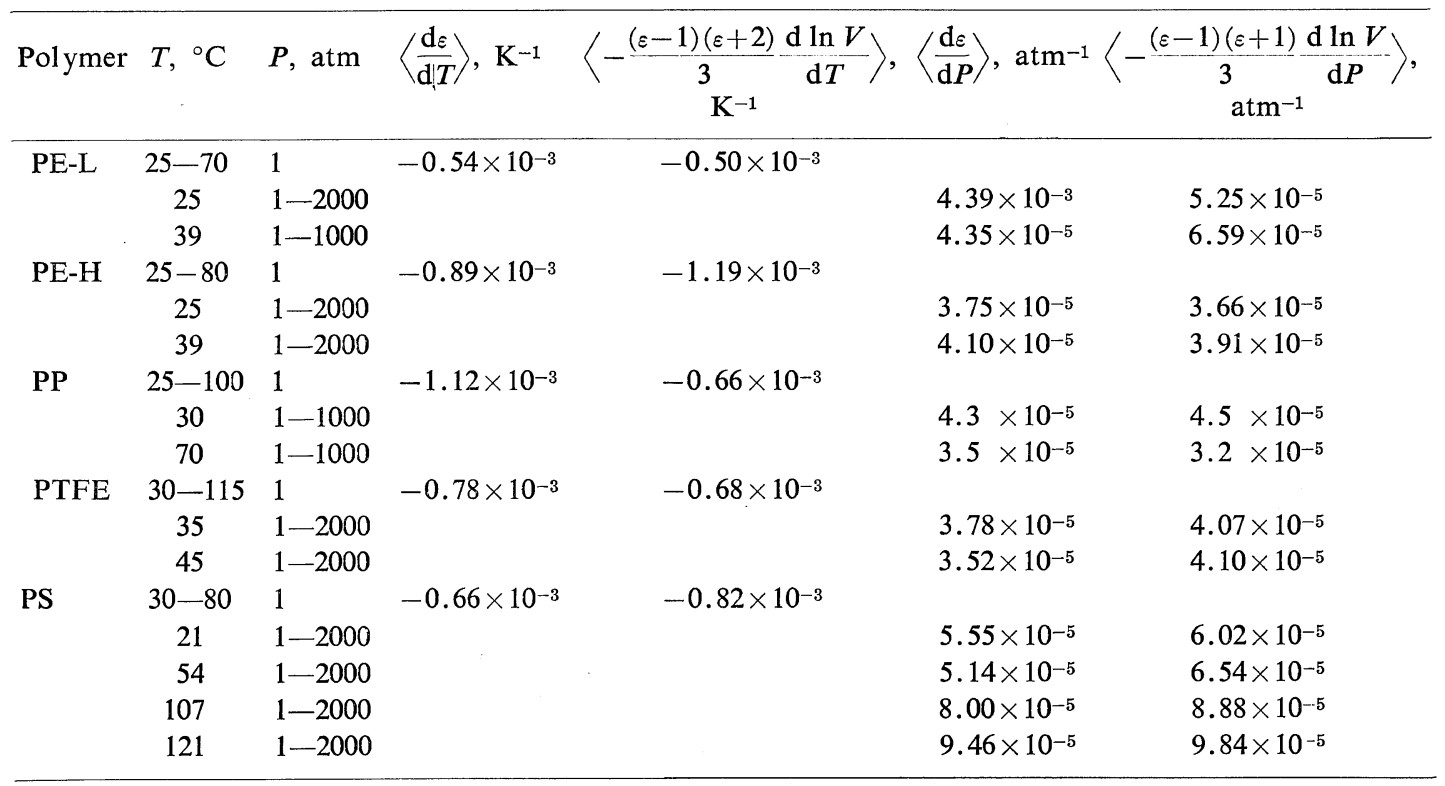

$(=1 / \rho)$. Table I shows that $\mathrm{d} \varepsilon / \mathrm{d} X$ is mainly determined by $\mathrm{d}(\ln V) / \mathrm{d} X$.

Relationship between $\alpha$ and Molecular Structure

Values of $\alpha$ would not be dominated by the state of aggregation of polymer chains, but by the molecular structure. This concept is confirmed in Figure 9. Figure 9 shows the comparison of the values of $C_{\mathrm{M}}$ (or $\alpha$ ) for polymers with those for low-molecular-weight substances whose molecular structures are similar to those 
of polymers. It is easily recognized that the value of $\alpha$ for the polymer shows close agreement with that for the corresponding low-molecular-weight substance, hence it would be concluded that the determining factor of $\alpha$ for a polymer is the molecular structure of the monomer unit. The value of $\alpha$ for $-\mathrm{CH}_{2}-\mathrm{CH}_{2}-$ unit is about two-thirds of that for $-\mathrm{CH}_{2}-\mathrm{CH}\left(\mathrm{CH}_{3}\right)$ unit. This fact indicates the difference in numbers of $\mathrm{C}$ and $\mathrm{H}$ atoms between the units, and it is reasonable if the additivity of $\alpha$ for the elementary units is assumed. For polar polymers the determining factor of $\varepsilon_{\infty}$ would be the molecular structure of a monomer unit as well. To confirm this concept, let's estimate the value of $\varepsilon_{\infty}$ for poly(vinylidene fluoride) (PVDF). According to the results shown in Figure $9, C_{\mathrm{M}}$ for $-\mathrm{CH}_{2}$ - unit (described as $C_{\mathrm{M}}$ $\left(-\mathrm{CH}_{2}-\right)$ hereafter) is about $4.9 \mathrm{~cm}^{3} \mathrm{~mol}^{-1}$ and $C_{\mathrm{M}}\left(-\mathrm{CF}_{2^{-}}\right)$is about $6.3 \mathrm{~cm}^{3} \mathrm{~mol}^{-1}$ ? Then $C_{\mathrm{M}}$ $\left(-\mathrm{CH}_{2}-\mathrm{CF}_{2}-\right)$ is estimated as $11.2 \mathrm{~cm}^{3} \mathrm{~mol}^{-1}$. As $M=64 \mathrm{~g} \mathrm{~mol}^{-1}$ and $\rho \simeq 1.8 \mathrm{~g} \mathrm{~cm}^{-3}$ for PVDF, $\varepsilon_{\infty}$ is evaluated as 2.38 ( $\left.\equiv \varepsilon_{\infty, \text { calc }}\right)$ from eq 1 . According to Bergmann, Jr., et al., ${ }^{7}$ the average refractive index $n$ of a PVDF film for the light of $\lambda=6328 \AA$ is 1.425 . This value gives the optical dielectric constant $\varepsilon_{\infty, \text { opt }}$ as $\varepsilon_{\infty, \text { opt }}=n^{2} \simeq$ 2.03. Since $\varepsilon_{\infty, \text { opt }}$ contains only a contribution of the optical polarization, $\varepsilon_{\infty, \text { opt }}$ is smaller than $\varepsilon_{\infty, \text { calc. }}$. In the previous work, ${ }^{8} \varepsilon$ of PVDF at $-49^{\circ} \mathrm{C}$ and $300 \mathrm{kHz}$ was found to be about 3.0. This value is considerably larger than $\varepsilon_{\infty, \text { calc. }}$ However, taking the fact that an effect of local-mode relaxation process on $\varepsilon$ still remains at this temperature and the frequency into consideration, we could conclude that $\varepsilon_{\infty}$ of PVDF is determined by the molecular structure of the monomer unit.

\section{Transition Phenomena}

It is easily recognized that the break points in Figures $1 \sim 8$ where $\partial \varepsilon / \partial T$ or $\partial \varepsilon / \partial P$ changes abruptly correspond to the transition points (or regions). That is, in Figures 2 and 7 this break point corresponds to the first crystalline transition point $T_{\chi}\left(20^{\circ} \mathrm{C}\right.$ transition $)$ of PTFE, and changes of $\varepsilon$ with $T$ and $P$ are caused by the discontinuous change in $V$ at this point (the first order transition). In Figures 3 and 8 it corresponds to the glass transition point $T_{\mathrm{g}}$ of



Figure 10. Pressure dependences of transition temperature in PS and PTFE.

PS (the second order transition). From Figures 7 and 8, pressure dependences of $T_{\chi}$ and $T_{\mathrm{g}}$ can be estimated. The result is shown in Figure 10. Values of $\mathrm{d} T_{\chi} / \mathrm{d} P$ for PTFE and $\mathrm{d} T_{\mathrm{g}} / \mathrm{d} P$ for PS are slightly larger than those obtained by other investigators. ${ }^{3,9}$ It should be noted that the present value of $\mathrm{d} T_{\chi} / \mathrm{d} P$ is determined from an $\varepsilon$ vs. $P$ relation at a fixed temperature, while $\mathrm{d} T_{\chi} / \mathrm{d} \boldsymbol{P}$ by Hirakawa, et al. ${ }^{9}$ was determined by an ultrasonic attenuation $v s$. $T$ relation at a fixed pressure.

\section{CONCLUSION}

Changes of the dielectric constant $\varepsilon$ in nonpolar polymers with temperature and pressure can be estimated from those of the density $\rho$ through the Clausius-Mossotti equation. From the comparison of $\alpha$ for low-molecular-weight substances with that for polymers it is concluded that the determining factor of $\alpha$ for polymers is a molecular structure of the monomer unit. In the transition regions, such as the glass transition, the partial melting, and the phase transition in crystalline regions, $\varepsilon$ is considerably 
sensitive to $T$ or $P$, and hence the pressure coefficient of the transition temperature, $\mathrm{d} T / \mathrm{d} P$, can be readily estimated.

\section{REFERENCES}

1. (a) J. M. O'Reilly, in "Modern Aspects of the Vitreous State', Vol. 3, J. D. Mackenzie, Ed., Butterworths, London, 1964, p 59; (b) S. Saito, H. Sasabe, T. Nakajima, and K. Yada, $J$. Polym. Sci., Part A-2, 6, 1297 (1968); (c) H. Sasabe and S. Saito, Polymer J., 3, 634 (1972).

2. W. G. S. Scaife, IEEE Conference Publication, 1970, No. 67, p 71.
3. K. H. Hellwege, W. Knappe, and P. Lehmann, Kolloid-Z. Z. Polym., 183, 110 (1962).

4. C. E. Weir, J. Res, Natl. Bur. Stand., 53, 245 (1954).

5. G. N. Foster, W. Waldman, and R. G. Griskey, Polym. Eng. Sci., 6, 131 (1966).

6. C. P. Smyth, in "Dielectric Behavior and Structure," McGraw Hill, New York N.Y., 1955, Chapter IX.

7. J. G. Bergmann, Jr., J.H. McFee, and G. R. Crane, Appl. Phys. Letters, 18, 203 (1971).

8. H. Sasabe, S. Saito, M. Asahina, and H. Kakutani, J. Polym. Sci., Part A-2, 7, 1405 (1969).

9. S. Hirakawa and T. Takemura, Japanese $J$. Appl. Phys., 7, 814 (1968). 